Please cite this article as:

A. Romero, C. Jiménez, J. González, P. López-Varo, M.J. Deen, J.A. Jiménez-Tejada, Compact modeling of the effects of illumination on the contact region of organic phototransistors, Organic Electronics, (2019), 70, 113-121.

(C2019. This manuscript version is made available under the CC-BY-NCND 4.0 license

http://creativecommons.org/licenses/by-nc-nd/4.0/

Digital Object Identifier:

10.1016/j.orgel.2019.04.008

Source:

https://www.sciencedirect.com/science/article/pii/S1566119919301739 


\title{
Compact modeling of the effects of illumination on the contact region of organic phototransistors
}

\author{
A. Romero ${ }^{\mathrm{a}, \mathrm{b}}$, C. Jiménez ${ }^{\mathrm{b}}$, J. González ${ }^{\mathrm{a}}$, P. López-Varo ${ }^{\mathrm{b}, \mathrm{c}}$, M. J. Deen ${ }^{\mathrm{d}}$, J. A. Jiménez-Tejada ${ }^{\mathrm{b}, *}$ \\ ${ }^{a}$ Departamento de Arquitectura y Tecnología de Computadores, CITIC-UGR, Universidad de Granada, Granada 18071, Spain \\ ${ }^{b}$ Departamento de Electrónica y Tecnología de los Computadores, CITIC-UGR, Universidad de Granada, Granada 18071, Spain \\ ${ }^{c}$ Institut Photovoltaïque d'Ile-de-France 30 RD 128, 91120 Palaiseau, France \\ ${ }^{d}$ Department of Electrical and Computer Engineering McMaster University, 1280 Main Street West, Hamilton, Ontario L8S 4K1, Canada
}

\begin{abstract}
A good modeling of degrading effects in an electronic device, such as the contact region of organic phototransistors (OPTs), can be favorably used to better describe and optimize the performance of the whole device. Furthermore, a proper design of the contacts can enhance the exciton dissociation and the extraction of photogenerated charge in the device. In this work, a compact model for OPTs is developed. This model is valid for all the operation regimes of the transistors. It includes a model for the contact region of the device that incorporates the effects of illumination. The compact model and the contact region model are validated with published experimental data from several OPTs under different illumination conditions. The tool used to validate the model is an evolutionary parameter extraction procedure developed in a previous work. The results show that both photoconductive and photovoltaic effects impact the intrinsic region of the transistor, as well as the electrical behavior of the contact region. The parameters used in the contact region model are linked to these photovoltaic and photoconductive effects.
\end{abstract}

Keywords: Phototransistors, thin-film transistor, device parameters, modeling, contact effects, many-objective optimization, multi-objective evolutionary algorithms, photovoltaic effect, photoconductive effect

\section{Introduction}

Over the past two decades, the organic (polymeric) semiconductor technology has attracted considerable research interest [1-3]. Many efforts have been made to develop different kinds of organic devices that cover a wide range of industrial and commercial applications, such as organic light-emitting diodes (LEDs), organic photovoltaic cells (OPVs) and organic thinfilm transistors (OTFTs) [1-3]. OTFTs are particularly interesting since they are the basis of other devices, such as memories and sensors.

Organic phototransistors (OPTs) are a particular case of transistors and sensors in which light detection and signal amplification are combined together in a single component. Like the rest of organic devices, OPTs show great advantages with regard to their inorganic counterparts, such as light-weight, lowcost, easy solution processing and flexibility [1]. Nevertheless, they also show common performance disadvantages, such as a low carrier mobility [2-4] or high process variability [5-9].

\footnotetext{
This work was supported by projects MAT2016-76892-C3-3-R and TIN2015-67020-P funded by the Spanish Government and European Regional Development Funds (ERDF) and by "Beca de Iniciación a la Investigación para estudiantes de Grado" given by the University of Granada.

${ }^{*}$ Corresponding author

Email addresses: ad90ry@correo.ugr.es (A. Romero), carmenjm186@correo.ugr.es (C. Jiménez), jesusgonzalez@ugr.es (J. González), pilar. lopez-varo@ipvf .fr (P. López-Varo), jamal@mcmaster.ca (M. J. Deen), tejada@ugr.es (J. A. Jiménez-Tejada)
}

Currently, the OPTs' photosensitivity and photoresponsivity are also inferior to those in inorganic phototransistors, although novel strategies are developed to enhance these properties, such as blending p-type and n-type semiconductors as donor-acceptor channel layers $[10,11]$. A key issue in the OPTs' performance is the contact quality at the semiconductor channel/electrode interfaces [12-15]. In fact, attempts to monitor the way the contact region in an OPT evolves with illumination were studied in the past [12,14, 16-18]. Contacts play a main role in the exciton dissociation and extraction of charge, which are essential mechanisms in OPTs' performance [19]. Also, contact effects degrade the performance of OTFTs. For this reason, they have been extensively studied and even incorporated in OTFT compact models [20-29]. However, from the authors' knowledge, the way the contact region evolves with illumination has not been introduced specifically in compact models for OPTs. The incorporation of this dependence of the contact region with illumination in a compact model would provide a better characterization of the device and would establish new optimization pathways. The resulting model would include more accurate values of the main parameters that control the current-voltage characteristics of the OPT: charge carrier mobility, threshold voltage and the contact resistance itself.

In order to fill this gap, a compact model that describes the current-voltage characteristics of OPTs is presented. The model includes the dependence of the voltage drop at the contact regions with illumination, and it is sensitive to the photoconductive and photovoltaic effects. In parallel with the model, a pa- 


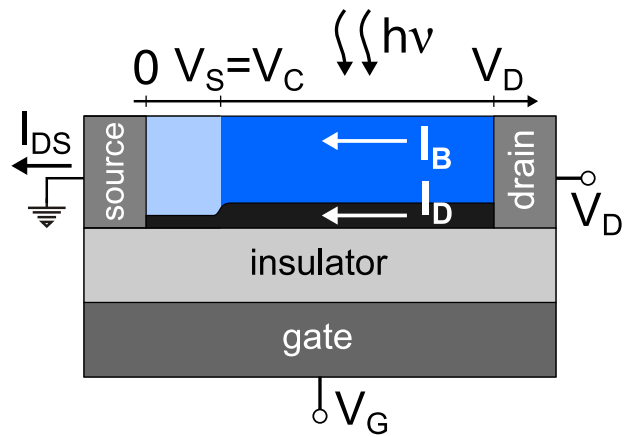

Figure 1: Cross-sectional view of an OPT structure with the bottom-contact configuration.

rameter extraction procedure is developed and implemented. It allows to check the validity of the model with experimental data.

This paper is organized as follows. In Section 2, the applicability of a widely known compact model in OTFTs including contact effects [25-27] is extended to OPTs, in which the photoconductive and photovoltaic mechanisms are incorporated in the model. In Section 3, a previously developed evolutionary parameter extraction procedure [30] is presented and adapted to the model. In Section 4, the proposed model is validated with published experimental data in OPTs under different illumination conditions. Finally, the conclusions are presented in Section 5.

\section{Theory}

Figure 1 shows a cross sectional view of an OPT structure. The source terminal voltage is zero and $V_{D}$ and $V_{G}$ are the drain and gate terminal voltages, respectively. $V_{S}$ (or $V_{C}$ ) is the voltage drop at the contact (the contact region is exaggerated for clarity). The electric current flowing between drain and source terminals $I_{D S}$ is composed of the field-effect (channel) current $I_{D}$ flowing in the accumulation layer and the bulk current $I_{B}$ [31]:

$$
I_{D S}=I_{B}+I_{D}
$$

In the depletion (cut-off) region, $I_{D} \ll I_{B}$ and $I_{D S} \simeq I_{B}$. In sub-threshold or over threshold conditions, $I_{B} \ll I_{D}$ and $I_{D S} \simeq$ $I_{D}$. In order to ensure a smooth transition from depletion to conduction, (1) can be written as:

$$
\begin{aligned}
& I_{D S}=I_{B}\left[1+\left(\frac{I_{D}}{I_{B}}\right)^{s}\right]^{1 / s}, \\
& \forall s \in \mathbb{Z}: s>0
\end{aligned}
$$

where $s$ is a free parameter employed to control the transition smoothness [32].

\subsection{Dark}

The objective of this work is to develop a compact model for the current-voltage characteristics of an OPT that incorporates the evolution of the contact region with the intensity of the light.
Since the OPT is a particular case of OTFT, then, as a starting point, we consider a well-established compact model for the OTFT [25, 26]:

$$
\begin{aligned}
& I_{D}=k^{\prime} \frac{W}{L} \frac{V_{E O D R}\left(V_{S}\right)^{(2+\gamma)}-V_{E O D R}\left(V_{D}\right)^{(2+\gamma)}}{2+\gamma} \\
& k^{\prime}=\mu_{0} C_{o x} ; V_{E O D R}(V)=V_{S S} \ln \left[1+\exp \left(\frac{V_{G}-V_{T}-V}{V_{S S}}\right)\right]
\end{aligned}
$$

which includes the voltage drop at the source contact, $V_{S} \equiv V_{C}$, and electric field-dependent mobility [33],

$$
\mu=\mu_{0}\left(V_{G}-V_{T}\right)^{\gamma},
$$

with $V_{T}$ being the threshold voltage, $C_{o x}$ the capacitance per unit area of the oxide, and $W$ and $L$ the channel width and length, respectively. $V_{S S}$ is a voltage parameter related to the steepness of the subthreshold characteristics of the TFT, $\gamma$ is the mobility enhancement factor, and $\mu_{0}$ is the mobility-related parameter, its dimension is expressed as $\mathrm{cm}^{2} /\left(\mathrm{V}^{1+\gamma} \mathrm{s}\right)$. In order to provide a single value for the voltage dependent mobility, the mobility is evaluated at $V_{G T}=V_{G}-V_{T}=1 \mathrm{~V}$, thus $\mu\left(V_{G T}=1\right.$ $\mathrm{V})=\mu_{0}$ in $\mathrm{cm}^{2} /(\mathrm{Vs})$. This compact model is able to describe all operation modes of the transistor: triode, saturation, subthreshold or even reverse biasing. The depletion region would require the use of (2).

In case the parameters $\gamma, V_{T}, V_{S S}$ and $k^{\prime}$ are known, the voltage in the contacts $V_{S}\left(I_{D}, V_{G}\right)$ would be extracted by introducing the values of the experimental data $\left(I_{D}, V_{G}, V_{D}\right)$ and the values of $\gamma, V_{T}, V_{S S}$ and $k^{\prime}$ in (3):

$$
\begin{aligned}
V_{S} & =V_{G}-V_{T}-V_{S S} \\
& \times \ln \left[\exp \left(\frac{\left(\frac{I_{D} L(\gamma+2)}{W k^{\prime}}+V_{E O D R}\left(V_{D}\right)^{(\gamma+2)}\right)^{\frac{1}{\gamma+2}}}{V_{S S}}\right)-1\right] .
\end{aligned}
$$

However, as these parameters are not known a priori, model (3) was lately redefined with the inclusion of a model for the contact region [27]:

$$
V_{S}=\left(\frac{I_{D}}{M_{C}}\right)^{\frac{1}{m_{k}}},
$$

where $m_{k}$ is a constant that indicates the grade of the observed trend (from linear to quadratic, $1 \leq m_{k} \leq 2$ ) in the triode region of the OTFT [34] and the parameter $M_{C}$ is usually gate voltage dependent:

$$
M_{C}=\alpha\left(V_{G}-V_{T}\right)^{(1+\gamma)}
$$

The subthreshold regime is not considered in (7), but it can be easily added by an asymptotically interpolation function, similar to that used in (3) for other field effect transistors [35-37]. The result is:

$$
M_{C}=\alpha V_{S S} \ln \left[1+\exp \left(\frac{V_{G}-V_{T}}{V_{S S}}\right)\right]^{1+\gamma} .
$$

$M_{C}$ coincides with the conductance of the contact region when $m_{k}=1$. The dependence of $M_{C}$ with the threshold voltage 
comes from two facts. The first one is through the gate voltage dependent carrier mobility (4), which is controlled with the parameter $\gamma$. The second one considers that the free charge density per unit area in the contact region $\sigma_{\text {contact }}$ is lower but proportional to the free charge density in the active channel $\sigma_{\text {channel }}$ $\left(\sigma_{\text {contact }}=\kappa \sigma_{\text {channel }}\right)$ with $\sigma_{\text {channel }}=C_{o x}\left(V_{G}-V_{T}\right)$. The proportionality between the free charge densities at these two adjacent regions is assumed, since there is no physical reason to believe that the mobile charges in these two regions start appearing at very different gate voltages, or follow very different trends, unless local non-uniformities were present just at the contact region. The value of the proportionality constant between these two charge densities $\kappa$, implicit in the value of $\alpha$, was tested to be sensitive to the contact material and temperature [27]. Overall, the application of the combined model (3), (6) and (7) can be found in different kinds of OTFTs [38-46]. Nevertheless, neither the model nor the sensitivity of the parameter $M_{C}$ to illumination were previously tested.

\subsection{Illumination}

The current-voltage characteristics of OPTs under illumination are controlled by the photoconductive and photovoltaic mechanisms. The photoconductive effect is detected when the transistor operates in the depletion region. The photovoltaic effect is observed in on-state and can be detected by a modification of the value of the threshold voltage. The extension of model for OPTs in dark (3)-(8) to illumination conditions needs to include the influence of the incident light $P$ on the parameters $I_{B}, V_{T}$ and $\alpha$.

\section{Bulk current, $I_{B}$}

In cut-off operation, the gate no longer controls the transistor and the device performs as an organic semiconductor sandwiched between two contacts. In this region, the photoresponse of the device is similar to that of photoresistors. The device current $I_{D S} \simeq I_{B}$, the photo-generated charge carriers enhance the conductivity of the bulk, and the current $I_{B}$ shows a linear increase with the optical power [12, 13, 16, 17, 47, 48]:

$$
I_{B}=\beta_{I} P+I_{B_{0}}
$$

where $I_{B_{0}}$ is the bulk current in dark and $\beta_{I}$ controls the linear increase of the bulk current with $P$.

\section{Threshold voltage, $V_{T}$}

When the OPT operates in conduction, including both subthreshold and over-threshold regions, the increase of the drain current with illumination is linked to a modification of the threshold voltage $\Delta V_{T}$. This modification can be associated to an accumulation of trapped photogenerated charges at the organic-insulator interface [49-51] or in layers close to the contact regions [52], with a high concentration of defects and traps, in which excitons are photogenerated and subsequently free charges are separated. In the semiconductor bulk, far from these layers and interfaces, the exciton recombination is high due to the absence of high electric fields. For this reason, recent OPT topologies involving bulk heterojunction (BHJ) composite of $\mathrm{p}$ type and n-type materials as channel layers provide additional dissociation sites for the photogenerated excitons [10,11]. The variation $\Delta V_{T}$ is usually modeled as [53,54]:

$$
V_{T}=V_{T d}-\Delta V_{T} ; \Delta V_{T}=\frac{A k T}{q} \ln \left(1+\frac{\eta q P}{I_{D d} h v}\right),
$$

where $V_{T d}$ and $I_{D d}$ are the threshold voltage and the channel current at dark conditions, respectively, $k$ is the Boltzmann constant, $T$ is the temperature, $q$ is the magnitude of the electron charge, $\eta$ is the quantum efficiency associated with the absorption process in the channel, $h v$ is the photon energy and $A$ is an empirical constant. The parameter $A$ was first introduced in [53] for studying High-Electron Mobility Transistors (HEMTs) under illumination. It was defined as the ratio of the variation of the threshold voltage in the transistor under illumination and the variation of the barrier seen by free carriers at the source. The lowering of the energy barrier at the source contact is attributed to an accumulation of trapped photogenerated carriers in this region, that was previously modeled for photodiodes [55].

It is remarkable how model (10), which initially was proposed as a link between the variation of the threshold voltage of the HEMT and the barrier lowering at the source region, has been applied successfully to their organic counterparts $[12,13,16,17,47,48,56,57]$. If such an important connection exists between the variation of the threshold voltage of the device and a possible barrier lowering at the contact region, then the transistor models should include the dependence of the contact region with illumination. In our model, it should be included in the contact conductance $M_{C}$.

\section{Contact conductance, $M_{C}$}

We mentioned above that the free charge density in the contact region is proportional to the free charge density in the channel $\left(\sigma_{\text {contact }}=\kappa \sigma_{\text {channel }}\right)$ and also lower than it. On the one hand, this means that both follow the same trend with $V_{G}-V_{T}$, and thus, the photovoltaic effect is implicit in the model of the contact region (6)-(8). On the other hand, the constant $\kappa$ being less than one means that there is margin for the conductivity of the contact region to be increased by modifying the temperature [27], the light intensity $P$, or any other physical or chemical external variable. Intuitively, since the bulk of the organic material is affected by the photoconductive effect, the contact region might also be affected. The photoconductive effect can be clearly distinguished in the cut-off region when the conducting channel and the photoconductive effect are negligible. However, this does not mean that the photoconductivity effect cannot affect other operating regions of the device. As the proportionality constant $\kappa$, which is implicit in $\alpha$ [27], relates the conductivity of the contact region and that of the channel, both $\kappa$ and $\alpha$ might be modulated by a linear function as described by the photoconductive effect. In this regard, the parameter $\alpha$ in (7)-(8) can be written as:

$$
\alpha=\beta_{\alpha} P+\alpha_{0},
$$

where $\alpha_{0}$ is the value of $\alpha$ in dark and $\beta_{\alpha}$ controls the linear evolution of $\alpha$ with the light intensity. 


\section{Evolutionary Parameter Extraction Procedure}

Once the model for OPTs is described, in which both photoconductive and photovoltaic effects are included in the contact model, a validation of this theory is necessary. For that purpose, output and transfer characteristics are evaluated with our model, aiming to reproduce published experimental measurements of OPTs. In order to compare experimental and numerical results, a parameter extraction procedure is necessary. Recently, the compact model for OTFTs in dark was complemented with a multi-objective [46] and a many-objective [30] evolutionary parameter extraction procedures. In this work, the many-objective evolutionary parameter extraction procedure is used in combination with an open source evolutionary tool called ECJ (A Java-based Evolutionary Computation Research System) [58].

Our procedure is based on a set of experimental data $I_{D}=I_{D}\left(V_{G_{i}}, V_{D_{j}}\right)$, where $i \in \mathbb{Z}: 1 \leq i \leq g$ and $j \in \mathbb{Z}: 1 \leq j \leq d$, and $g$ and $d$ are the total number of discrete values of $V_{G}$ and $V_{D}$, respectively. The numerical estimation of $I_{D}$ with (3) and (6), and the numerical estimation of the voltage drop at the contact region $V_{S}$ with (5) are named $\widehat{I_{D}}\left(V_{G_{i}}, V_{D_{j}}, x\right)$ and $\widehat{V_{S}}\left(V_{G_{i}}, V_{D_{j}}, x\right)$, respectively, where $x$ refers to the set of parameters needed to compute (3)-(7), and is defined as an individual of the population:

$$
x=\left(k^{\prime}, \gamma, V_{T}, V_{S S}, m_{k}, M_{C}\left(V_{G_{1}}\right), \ldots, M_{C}\left(V_{G_{g}}\right)\right)
$$

where $x_{l}(l \in \mathbb{Z}: 1 \leq l \leq p)$ is a variable of an individual and $p=g+5$ is the length or number of variables of an individual (12).

The evolutionary parameter extraction procedure solves a many-objective problem (MaOP). In our MaOP, four objectives have been defined for an individual (12):

$\left(O_{1}\right)$ to minimize the error between the experimental values of $I_{D}\left(V_{G_{i}}, V_{D_{j}}\right)$ and their estimation from (3) and (6) $\widehat{I_{D}}\left(V_{G_{i}}, V_{D_{j}}, x\right)$;

$\left(O_{2}\right)$ to minimize the error between the voltage drops at the contact region $V_{S}\left(V_{G_{i}}, V_{D_{j}}, x\right)$ described by (6) and their estimation $\widehat{V_{S}}\left(V_{G_{i}}, V_{D_{j}}, x\right)$ extracted from (5);

$\left(\mathrm{O}_{3}\right)$ to maximize the standard determination coefficient $R^{2}\left(V_{G_{i}}, x\right)$ of the linear fit of $M_{C}^{1 /(1+\gamma)}$ with $V_{G}$, which extracted from (7) is:

$$
M_{C}{ }^{1 /(1+\gamma)}\left(V_{G}\right)=\alpha^{1 /(1+\gamma)} V_{G}-\alpha^{1 /(1+\gamma)} \hat{V}_{T} ;
$$

and $\left(O_{4}\right)$ to minimize the difference between $V_{T}$ coded in $x$ $\left(x_{3}\right)$ and its estimation $\widehat{V_{T}}\left(V_{G_{i}}, x\right)$ extracted from the linear fit (13).

The Normalized Root Mean Squared Error (NRMSE) is used to estimate errors $\left(O_{1}\right)$ and $\left(O_{2}\right)[59]$ :

$$
\operatorname{NRMSE}(y, \hat{y})=\sqrt{\frac{\sum_{z=1}^{w}\left(y_{z}-\hat{y}_{z}\right)^{2}}{\sum_{z=1}^{w}\left(y_{z}-\bar{y}\right)^{2}}}
$$

where $y$ represents the data set that we want to accurately approximate, $\hat{y}$ is the estimation of $y, w$ is the number of data samples in $y$, and $\bar{y}$ is the mean value of the complete data set $y$.
Thus our minimization MaOP, named $O$, is defined as $O=$ $\left(\mathrm{O}_{1}, \mathrm{O}_{2}, \mathrm{O}_{3}, \mathrm{O}_{4}\right)$, where

$$
\begin{aligned}
& O_{1}(x)= \operatorname{NRMSE}\left(I_{D}\left(V_{G_{i}}, V_{D_{j}}\right), \widehat{I_{D}}\left(V_{G_{i}}, V_{D_{j}}, x\right)\right), \\
& O_{2}(x)= \operatorname{NRMSE}\left(V_{S}\left(V_{G_{i}}, V_{D_{j}}, x\right), \widehat{V_{S}}\left(V_{G_{i}}, V_{D_{j}}, x\right)\right), \\
& O_{3}(x)= 1-R^{2}\left(V_{G_{i}}, x\right), \\
& O_{4}(x)=\left|\widehat{V_{T}}\left(V_{G_{i}}, x\right)-x_{3}\right|=\left|\widehat{V_{T}}\left(V_{G_{i}}, x\right)-V_{T}\right|, \\
& \\
& \forall i \in \mathbb{Z}: 1 \leq i \leq g, \forall j \in \mathbb{Z}: 1 \leq j \leq d
\end{aligned}
$$

Objective $\left(O_{1}\right)$ allows us to accurately reproduce the experimental output and transfer characteristics with our model, using the parameters coded in (12). Objective $\left(O_{2}\right)$ checks whether the trend of the $I_{D}-V_{S}$ curves extracted from (5) along with the parameters coded in (12) are physically valid. Objectives $\left(\mathrm{O}_{3}\right)$ and $\left(\mathrm{O}_{4}\right)$ are not mandatory [46], but serve as a guide in the search process to those solutions that approach to the trend given in (7).

Along with the MaOP definition (15), the procedure is composed by some simple constraints (other constraints are implicit in the search space definition) to converge to physically acceptable solutions:

(i) $\widehat{V_{S}}\left(V_{G_{i}}, V_{D_{j}}, x\right) \geq 0$

(ii) $M_{C}\left(V_{G_{i}}\right)>0$.

A detailed explanation of our evolutionary parameter extraction procedure can be found in [30].

\section{Results}

Our model for OTFTs under illumination is now tested with two sets of experimental data from different OPTs [17, 56]. Output and transfer characteristics were measured at different light intensities in both sets of transistors. The procedure to analyze the two OPTs is the same and is detailed below.

In the two cases, every set of output characteristics $\left(I_{D}-V_{D}\right.$ curves), measured at a different illumination intensity, is analyzed separately from the rest. First, the values of the parameters included in an individual of the population (12), that makes model (3)-(7) fulfill objectives $\left(O_{1}\right),\left(O_{2}\right),\left(O_{3}\right)$ and $\left(O_{4}\right)$, are extracted. Note (i) that the extraction procedure is independently applied to each set of experimental $I_{D}-V_{D}$ curves; (ii) that equations (3)-(7) alone does not include any information about the light intensity; and (iii) that equations (10)-(11) are not used in the extraction procedure. The resulting values will be shown in a Table. Second, the values of the parameters $V_{T}, \alpha$ and $M_{C}\left(V_{G_{i}}\right)$ are represented as a function of the light intensity. Then, we will check whether the evolution of these parameters with light intensity follow the trend proposed in (10)-(11). As a final test, the results of the model are compared with transfer curves measured at different light intensities. 
Table 1: Parameter values of the NBBI OPT under different illumination conditions $\left(k^{\prime}\right.$ is in $\mathrm{A} / \mathrm{V}^{2+\gamma}, V_{T}$ and $V_{S S}$ in $\mathrm{V}$ and $M_{C}\left(V_{G_{i}}\right)$ in $\left.\mathrm{A} / \mathrm{V}^{m_{k}}\right)$.

\begin{tabular}{lcccc}
\hline $\mathrm{x}$ & dark & $50 \mathrm{~mW} / \mathrm{cm}^{2}$ & $70 \mathrm{~mW} / \mathrm{cm}^{2}$ & $80 \mathrm{~mW} / \mathrm{cm}^{2}$ \\
\hline$k^{\prime}$ & $7.25 \times 10^{-12}$ & $3.22 \times 10^{-12}$ & $2.42 \times 10^{-12}$ & $2.29 \times 10^{-12}$ \\
$\gamma$ & $2.50 \times 10^{-1}$ & $2.50 \times 10^{-1}$ & $2.50 \times 10^{-1}$ & $2.50 \times 10^{-1}$ \\
$V_{T}$ & $8.57 \times 10^{+0}$ & $-3.21 \times 10^{+1}$ & $-5.40 \times 10^{+1}$ & $-6.58 \times 10^{+1}$ \\
$V_{S S}$ & $2.59 \times 10^{+1}$ & $2.85 \times 10^{+1}$ & $2.52 \times 10^{+1}$ & $2.87 \times 10^{+1}$ \\
$m_{k}$ & $1.00 \times 10^{+0}$ & $1.00 \times 10^{+0}$ & $1.00 \times 10^{+0}$ & $1.00 \times 10^{+0}$ \\
$M_{C}(20 \mathrm{~V})$ & $5.98 \times 10^{-9}$ & $3.43 \times 10^{-8}$ & - & $6.87 \times 10^{-8}$ \\
$M_{C}(30 \mathrm{~V})$ & $9.68 \times 10^{-9}$ & $4.57 \times 10^{-8}$ & - & $8.30 \times 10^{-8}$ \\
$M_{C}(40 \mathrm{~V})$ & $1.56 \times 10^{-8}$ & $5.66 \times 10^{-8}$ & $7.79 \times 10^{-8}$ & $9.37 \times 10^{-8}$ \\
$M_{C}(50 \mathrm{~V})$ & $2.34 \times 10^{-8}$ & $6.64 \times 10^{-8}$ & $8.65 \times 10^{-8}$ & $1.03 \times 10^{-7}$ \\
$M_{C}(60 \mathrm{~V})$ & $3.22 \times 10^{-8}$ & $7.62 \times 10^{-8}$ & $9.46 \times 10^{-8}$ & $1.13 \times 10^{-7}$ \\
$M_{C}(70 \mathrm{~V})$ & $4.14 \times 10^{-8}$ & $8.37 \times 10^{-8}$ & $1.07 \times 10^{-7}$ & $1.25 \times 10^{-7}$ \\
$M_{C}(80 \mathrm{~V})$ & $5.06 \times 10^{-8}$ & $9.27 \times 10^{-8}$ & $1.21 \times 10^{-7}$ & $1.36 \times 10^{-7}$ \\
\hline
\end{tabular}
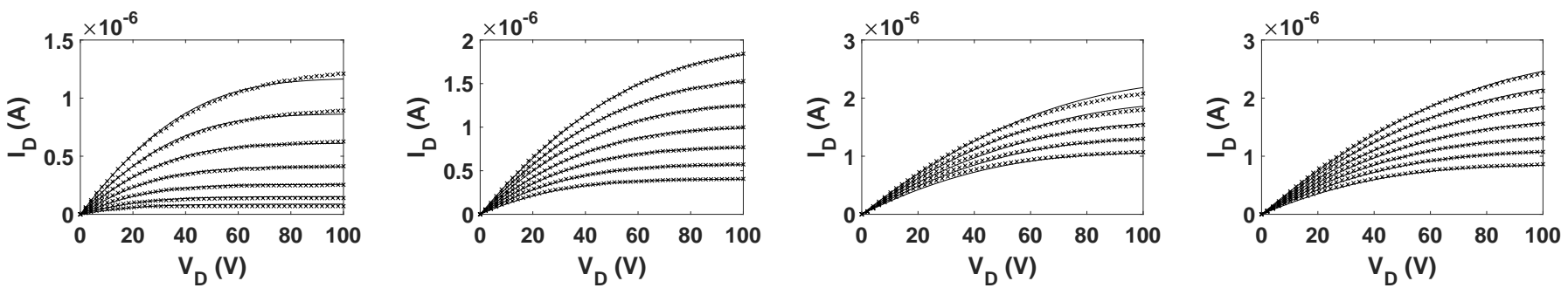

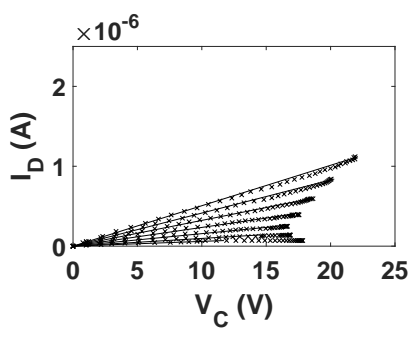

(a)

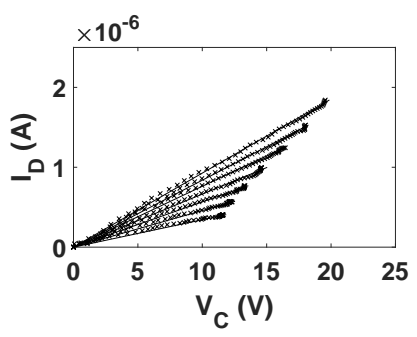

(b)

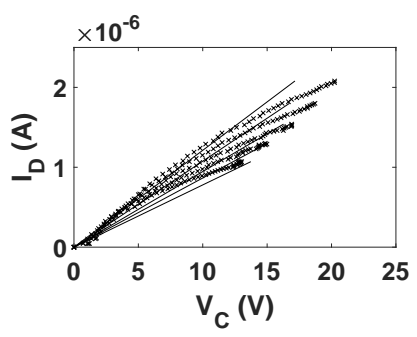

(c)

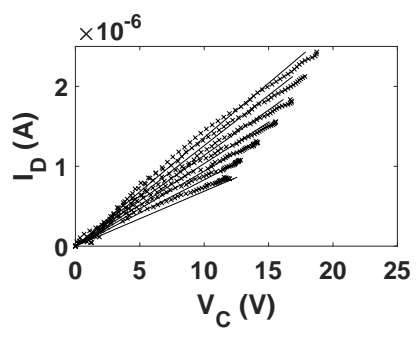

(d)

Figure 2: Output characteristics and contact $I_{D}-V_{C}$ curves in NBBI OPTs: (a) under dark, and under illumination at (b) $50 \mathrm{~mW} / \mathrm{cm}^{2}$, (c) $70 \mathrm{~mW} / \mathrm{cm}^{2}$ and (d) $80 \mathrm{~mW} / \mathrm{cm}^{2}$. Top side: Comparison of experimental $I_{D}-V_{D}$ curves $(\times)$ and our calculations with (3) (solid lines). Bottom side: Comparison of $I_{D}-V_{C}$ curves calculated with (5) $(\times)$ and (6) (solid lines). In (a), (b) and (d), $V_{G}$ is swept from 20 (bottom) to $80 \mathrm{~V}$ (top) with a $10 \mathrm{~V}$ step. In (c), $V_{G}$ is swept from 40 (bottom) to $80 \mathrm{~V}$ (top) with a $10 \mathrm{~V}$ step.

\subsection{NNBI OPT}

The first set of experimental data correspond to a naphthalene bis-benzimidazole (NBBI) based OTFT designed as a phototransistor [17]. The output and transfer characteristics measured by the authors under dark and at 50,70 and $80 \mathrm{~mW} / \mathrm{cm}^{2}$ white light intensities [17] are represented with symbols in Figs. 2 (top side) and 3, respectively. The transistor was fabricated with a top contact/bottom gate geometry in order to detect visible light. The substrate was an indium tin oxide (ITO) coated glass, which was patterned by etching with diluted $\mathrm{HCl}$ for a bottom gate electrode. The gate insulator layer was made of divinyl tetramethyl disiloxane-bis (benzo-cyclobutene) (BCB) with a capacitance per unit area $C_{o x}=1.73 \mathrm{nF} / \mathrm{cm}^{2}$. The $60 \mathrm{~nm}$ Aluminum (Al) (purity 99.9\%) source and drain electrodes were deposited by thermal evaporation under high vacuum $\left(2 \times 10^{-6}\right.$ mbar $)$ to obtain high quality ohmic contacts with the NBBI thin films. The channel length is $L=40 \mu \mathrm{m}$ and the channel width $W=2000 \mu \mathrm{m}$. The full protocols of the electrode deposition, substrate cleaning and surface passivation were given in Ref. [17, 60]. All current-voltage characteristics of the fabricated OTFTs were measured in an inert gas atmosphere.

Each set of $I_{D}-V_{D}$ curves of Fig. 2, corresponding to the four different illumination intensities, is analyzed. The values of the parameters included in an individual of the population (12), that makes model (3)-(7) fulfill objectives $\left(O_{1}\right),\left(O_{2}\right),\left(O_{3}\right)$ and $\left(O_{4}\right)$ are extracted and shown in Table 1 . Figure 2 shows the best fittings of objectives $\left(\mathrm{O}_{1}\right)$ and $\left(\mathrm{O}_{2}\right)$. The carrier mobility used in the fitting is $\mu_{0}=4.2 \times 10^{-3}, 1.9 \times 10^{-3}, 1.4 \times 10^{-3}, 1.3 \times 10^{-3}$ $\mathrm{cm}^{2} / \mathrm{Vs}$ under dark and at 50,70 and $80 \mathrm{~mW} / \mathrm{cm}^{2}$ respectively. The values of the carrier mobility extracted with our model are consistent with those obtained in [17]. Our model, which in- 


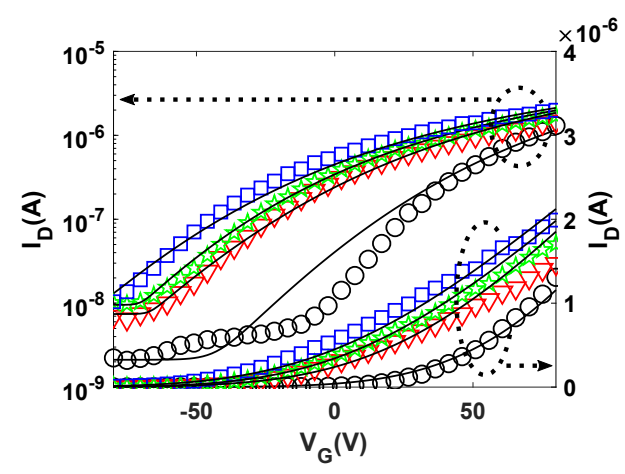

Figure 3: Comparison of experimental (symbols) transfer characteristics in saturation regime $\left(V_{D}=80 \mathrm{~V}\right)$ of NBBI OPTs, and our calculations with (3) (solid lines). Symbols: dark (black circles), illumination at $50 \mathrm{~mW} / \mathrm{cm}^{2}$ (red triangles), $70 \mathrm{~mW} / \mathrm{cm}^{2}$ (green stars) and $80 \mathrm{~mW} / \mathrm{cm}^{2}$ (blue squares). Note that the left $y$-axis is a log scale and the right $y$-axis is a linear scale for the same $I_{D}$ values.

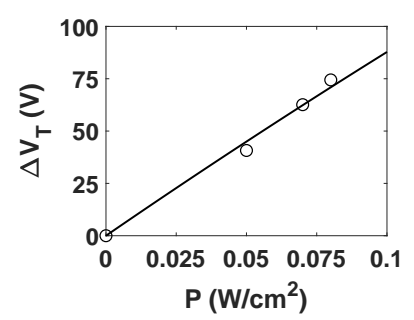

(a)

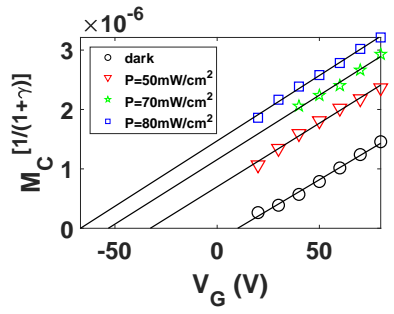

(b)

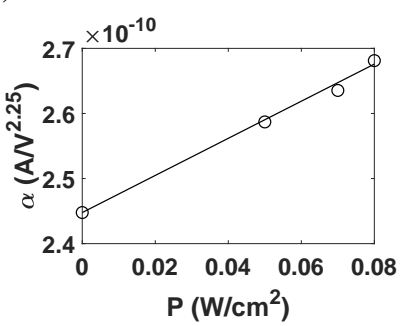

(c)

Figure 4: (a) Extracted values of $\Delta V_{T}$ (circles) and the trend of $\Delta V_{T}$ with illumination according to (10) (solid line). (b) Extracted values of $M_{C}\left(V_{G_{i}}\right)^{1 /(1+\gamma)}$ (symbols) and its fitting with (13) (solid lines). (c) Extracted values of $\alpha$ from (13) (circles) and the trend of $\alpha$ with illumination according to (11) (solid line) for the NBBI OPT.

cludes the contact effects, provides more realistic values than those obtained with the ideal MOS model [40, 46]. Also note that the value of the mobility $\mu_{0}$ decreases slightly from dark to illumination conditions as observed in [49, Fig. 6]. This slight decrease can be linked to the increment of charge carrier concentration with illumination. At high charge carrier concentrations, the effect of the energetic disorder disappears and the mobility decreases slightly due to the lower density of unoccupied states available for the hopping transport [61].

The extracted values of the parameters $V_{T}, M_{C}\left(V_{G_{i}}\right)$ and $\alpha$ are represented with symbols as a function of the light intensity in Fig. 4a, Fig. 4b and Fig. 4c, respectively. The solid line in Fig. 4a represents the fitting with (10) with $A=3.46 \times 10^{4}$, $\eta / I_{D d}=2.57 \mathrm{~A}^{-1}, h v=2.48 \mathrm{eV}$. Fig. $4 \mathrm{~b}$ shows the fulfillment of objectives $\left(\mathrm{O}_{3}\right)$ and $\left(\mathrm{O}_{4}\right)$. The solid lines in Fig. 4b are the fittings with (13), in which the values of $\hat{V}_{T}$ are practically the same to those found in Table 1. On average, the error between the values of $V_{T}$ and $\hat{V}_{T}$ is less than $5.5 \%$. Finally, the solid line of Fig. $4 \mathrm{c}$ represents the fitting with (11) using $\beta_{\alpha}=2.85 \times 10^{-10}$ $\mathrm{A} \mathrm{cm} /\left(\mathrm{V}^{2.25} \mathrm{~W}\right)$ and $\alpha_{0}=2.45 \times 10^{-10} \mathrm{~A} / \mathrm{V}^{2.25}$.

The good agreement shown in Fig. 2 between the experimental (symbols) and estimated (solid lines) $I_{D}-V_{D}$ curves is corroborated in Fig. 3 with a good fitting of experimental transfer curves measured at different illumination conditions (symbols) and our estimation (solid lines).

\subsection{EHPDI OPT}

A second test of our model is done with [N,N'-di(2ethylhexyl)-3,4,9,10-perylene diimide] (EHPDI) based OPTs [56] with a top contact/bottom gate geometry and BCB as gate insulator with a capacitance per unit area $C_{o x}=1.73 \mathrm{nF} / \mathrm{cm}^{2}$. The output and transfer characteristics, measured by the authors under dark and at 70, 79.2 and $91.06 \mathrm{~mW} / \mathrm{cm}^{2}$ white light intensities [56], are represented with crosses in Fig. 5 (top side) and Fig. 6, respectively. Al metal electrodes (purity 99.9\%) with a thickness $60 \mathrm{~nm}$ were deposited on the EHPDI active layer by using the vacuum evaporator under $2 \times 10^{-6} \mathrm{mbar}$. The channel length is $L=40 \mu \mathrm{m}$ and the channel width $W=2000 \mu \mathrm{m}$. The full protocols of the substrate cleaning, and surface passivation were given in Ref. [56]. The electrical characterization of the fabricated device was conducted in an inert gas atmosphere inside a glove box.

The evolutionary procedure is applied to each set of $I_{D}-V_{D}$ curves of Fig. 5, corresponding to the four different illumination intensities. The values of the parameters used in the calculation to fulfill objectives $\left(\mathrm{O}_{1}\right),\left(\mathrm{O}_{2}\right),\left(\mathrm{O}_{3}\right)$ and $\left(\mathrm{O}_{4}\right)$ are extracted and shown in Table 2. Figure 5 shows the best fittings of objectives $\left(O_{1}\right)$ and $\left(O_{2}\right)$, in which a good agreement between the experimental (symbols) and calculated $I_{D}-V_{D}$ curves (lines) is observed and the extracted contact curves $I_{D}-V_{C}$ (symbols) show a good physical meaning.

Once again, the good agreement observed in Fig. 5 between the experimental (symbols) and calculated (solid lines) $I_{D}-V_{D}$ curves is reinforced with the fitting of transfer $I_{D}-V_{G}$ curves in Fig. 6 (symbols for the experimental data and solid lines for our calculations). The filled symbols correspond to values taken from the output characteristics. These filled symbols are represented to indicate possible errors in the measurements due to factors not considered in the model, such as dynamic or hysteresis effects. It is clear that our calculations fit better the values taken from the output characteristics, in which the extraction procedure is carried out. In any case, it is remarkable how the whole experimental $I_{D}-V_{G}$ curves are reproduced with our calculations, including the one shown with diamonds in Fig. 6(a), which was measured under illumination at $56.4 \mathrm{~mW} / \mathrm{cm}^{2}$ (note that no output characteristics were analyzed at this illumination intensity).

The carrier mobility used in the fittings is $\mu_{0}=2.4 \times 10^{-3}$, $6.1 \times 10^{-4}, 6.0 \times 10^{-4}, 5.9 \times 10^{-4} \mathrm{~cm}^{2} /$ Vs under dark and at 70 , 79.2 and $91.06 \mathrm{~mW} / \mathrm{cm}^{2}$, respectively. These values are determined at $V_{G T}=1 \mathrm{~V}$. At other values of $V_{G T}$, the mobility can 
Table 2: Parameter values of the EHPDI OPT $\left(k^{\prime}\right.$ is in $\mathrm{A} / \mathrm{V}^{2+\gamma}, V_{T}$ and $V_{S S}$ in $\mathrm{V}$ and $M_{C}\left(V_{G}\right)$ in $\left.\mathrm{A} / \mathrm{V}^{m_{k}}\right)$ under different illumination conditions.

\begin{tabular}{lcccc}
\hline $\mathrm{x}$ & dark & $70 \mathrm{~mW} / \mathrm{cm}^{2}$ & $79.2 \mathrm{~mW} / \mathrm{cm}^{2}$ & $91.06 \mathrm{~mW} / \mathrm{cm}^{2}$ \\
\hline$k^{\prime}$ & $4.14 \times 10^{-12}$ & $1.04 \times 10^{-12}$ & $1.04 \times 10^{-12}$ & $1.01 \times 10^{-12}$ \\
$\gamma$ & $2.50 \times 10^{-1}$ & $2.50 \times 10^{-1}$ & $2.50 \times 10^{-1}$ & $2.50 \times 10^{-1}$ \\
$V_{T}$ & $1.23 \times 10^{+1}$ & $-6.81 \times 10^{+1}$ & $-7.06 \times 10^{+1}$ & $-7.07 \times 10^{+1}$ \\
$V_{S S}$ & $1.70 \times 10^{+1}$ & $2.06 \times 10^{+1}$ & $2.26 \times 10^{+1}$ & $1.94 \times 10^{+1}$ \\
$m_{k}$ & $1.00 \times 10^{+0}$ & $1.00 \times 10^{+0}$ & $1.00 \times 10^{+0}$ & $1.00 \times 10^{+0}$ \\
$M_{C}(20 \mathrm{~V})$ & $6.08 \times 10^{-10}$ & $2.77 \times 10^{-8}$ & $2.97 \times 10^{-8}$ & $3.37 \times 10^{-8}$ \\
$M_{C}(30 \mathrm{~V})$ & $1.40 \times 10^{-9}$ & $3.30 \times 10^{-8}$ & $3.47 \times 10^{-8}$ & $3.91 \times 10^{-8}$ \\
$M_{C}(40 \mathrm{~V})$ & $2.33 \times 10^{-9}$ & $3.68 \times 10^{-8}$ & $3.95 \times 10^{-8}$ & $4.41 \times 10^{-8}$ \\
$M_{C}(50 \mathrm{~V})$ & $3.36 \times 10^{-9}$ & $4.01 \times 10^{-8}$ & $4.28 \times 10^{-8}$ & $4.82 \times 10^{-8}$ \\
$M_{C}(60 \mathrm{~V})$ & $4.69 \times 10^{-9}$ & $4.52 \times 10^{-8}$ & $4.74 \times 10^{-8}$ & $5.36 \times 10^{-8}$ \\
$M_{C}(70 \mathrm{~V})$ & $6.19 \times 10^{-9}$ & $4.92 \times 10^{-8}$ & $5.20 \times 10^{-8}$ & $5.83 \times 10^{-8}$ \\
$M_{C}(80 \mathrm{~V})$ & $7.60 \times 10^{-9}$ & $5.43 \times 10^{-8}$ & $5.70 \times 10^{-8}$ & $6.47 \times 10^{-8}$ \\
\hline
\end{tabular}
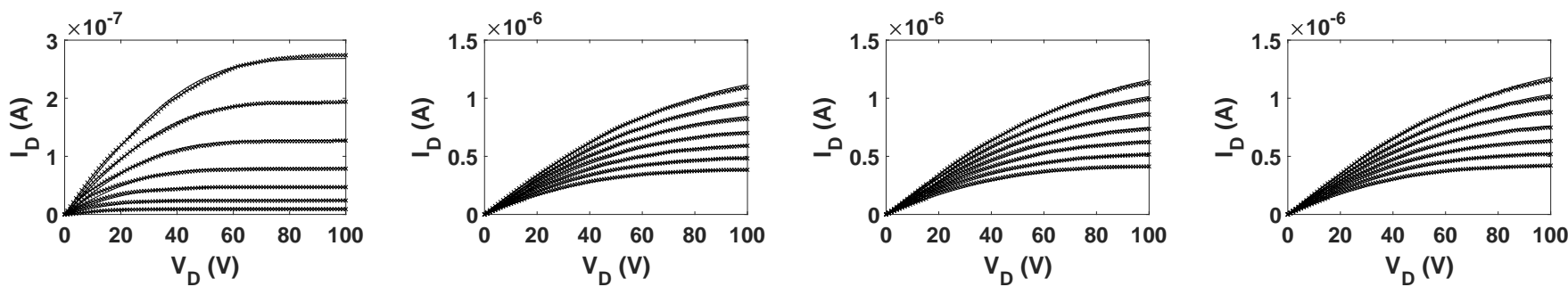

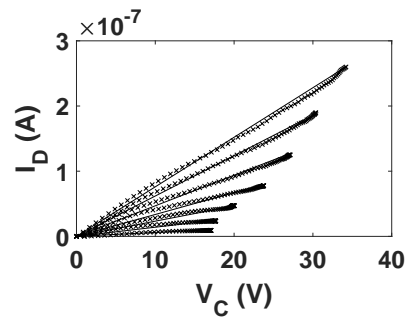

(a)

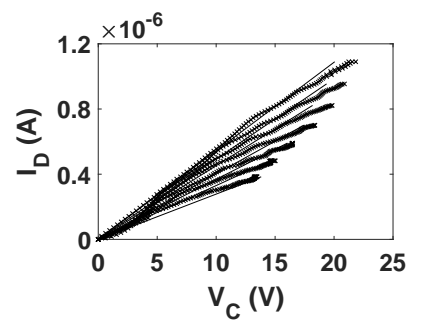

(b)

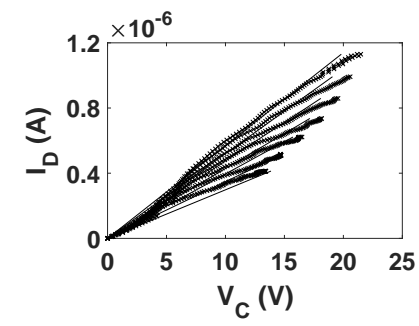

(c)

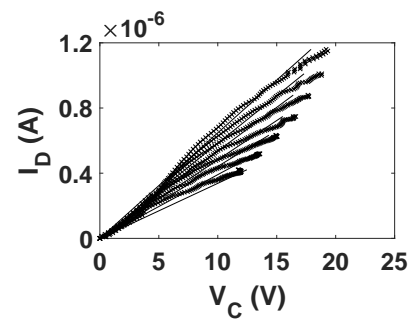

(d)

Figure 5: Output characteristics and contact $I_{D}-V_{C}$ curves in EHPDI OPTs: (a) under dark, and under illumination at (b) $70 \mathrm{~mW} / \mathrm{cm}^{2}$, (c) $79.2 \mathrm{~mW} / \mathrm{cm}^{2}$ and (d) $91.06 \mathrm{~mW} / \mathrm{cm}^{2}$. Top side: Comparison of experimental $I_{D}-V_{D}$ curves $(\times)$ and our calculations with (3) (solid lines). Bottom side: Comparison of $I_{D}-V_{C}$ curves calculated with (5) $\left(\times\right.$ ) and (6) (solid lines). In (a)-(d), $V_{G}$ is swept from 20 (bottom) to $80 \mathrm{~V}$ (top) with a $10 \mathrm{~V}$ step.

increase one order of magnitude, in agreement with values obtained for other PDI based polymers [62, 63]. Again, a decrease of $\mu_{0}$ with $P$ is observed.

The extracted values of the parameters $V_{T}, M_{C}\left(V_{G_{i}}\right)$ and $\alpha$ are represented with symbols as a function of the light intensity in Fig. 7a, Fig. 7b and Fig. 7c, respectively. The solid line in Fig. 7a represents the fitting with (10) with $A=4.41 \times 10^{2}, \eta / I_{D d}=$ $4.28 \times 10^{4} \mathrm{~A}^{-1}, h v=2.48 \mathrm{eV}$. Fig. 7b shows the fulfillment of objectives $\left(\mathrm{O}_{3}\right)$ and $\left(\mathrm{O}_{4}\right)$. The solid lines in Fig. $7 \mathrm{~b}$ are the fittings with (13), in which the values of $\hat{V}_{T}$ are practically the same to those found in Table 2. On average, the error between the values of $V_{T}$ and $\hat{V}_{T}$ is less than $0.3 \%$. Finally, The solid line of Fig. 7c represents the fitting with (11) using $\beta_{\alpha}=9.30 \times 10^{-10}$ $\mathrm{A} \mathrm{cm} /\left(\mathrm{V}^{2.25} \mathrm{~W}\right)$ and $\alpha_{0}=3.84 \times 10^{-11} \mathrm{~A} / \mathrm{V}^{2.25}$.

\subsection{Discussion}

The excellent fittings of Figs. $4 a, 4 b$ and $4 c$, for the NBBI transistor, and of Figs. 7a, 7b and 7c, for the EHPDI transistor, show that the values extracted for the parameters $V_{T}, M_{C}\left(V_{G_{i}}\right)$ and $\alpha$, obtained separately for each value of the illumination intensity, follow the trends predicted with our model. These fittings clearly indicate that the contact region of the phototransistor is highly sensitive to the photoconductive and photovoltaic effects.

The photovoltaic effect controls the electrical performance of both the intrinsic channel and the contact region when an accumulation channel exists along the transistor. It is detected with changes in the value of the threshold voltage. The photoconductive effect controls the conductivity of the contact and bulk regions of the semiconductor. Formerly, it was detected in the cut-off region by measuring the remnant current $I_{B}$. In our work, we have seen that it can also be detected analyzing the evolution of parameter $\alpha$ with the light intensity. In order to quantify how the photoconductive effect affects the contact region, we determine the value of the contact resistance at the 


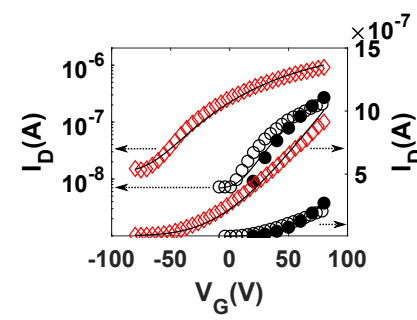

(a)

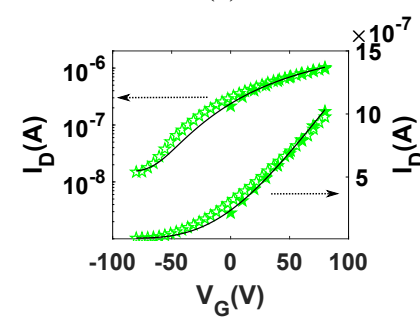

(c)

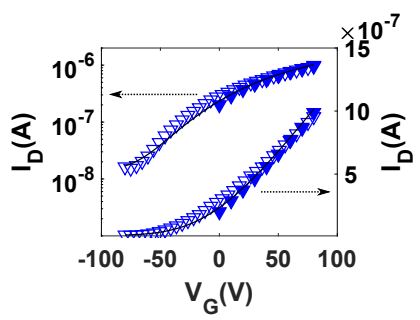

(b)

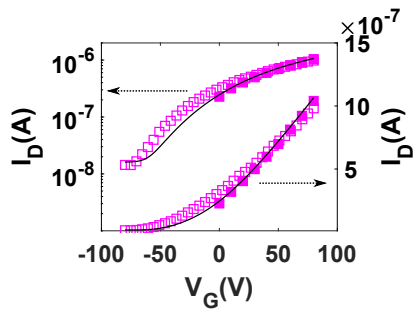

(d)
Figure 6: Comparison of experimental transfer characteristics in saturation regime $\left(V_{D}=80 \mathrm{~V}\right)$ of EHPDI OPTs (symbols) and our calculations with (3) (solid lines): (a) under dark (circles) and under illumination at $56.4 \mathrm{~mW} / \mathrm{cm}^{2}$ (diamonds), (b) $70 \mathrm{~mW} / \mathrm{cm}^{2}$, (c) $79.2 \mathrm{~mW} / \mathrm{cm}^{2}$ and (d) $91.06 \mathrm{~mW} / \mathrm{cm}^{2}$. The filled symbols correspond to values taken from the output characteristics.

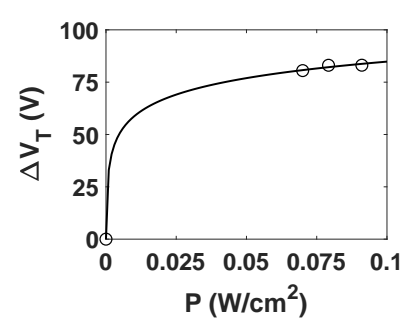

(a)

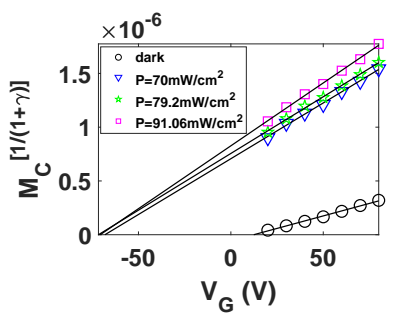

(b)

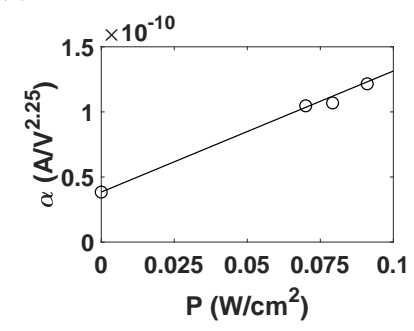

(c)

Figure 7: (a) Extracted values of $\Delta V_{T}$ (circles) and the trend of $\Delta V_{T}$ with illumination according to (10) (solid line). (b) Extracted values of $M_{C}\left(V_{G_{i}}\right)^{1 /(1+\gamma)}$ (symbols) and its fitting with (13) (solid lines). (c) Extracted values of $\alpha$ from (13) (circles) and the trend of $\alpha$ with illumination according to (11) (solid line) for the EHPDI OPT.

cut-off region from (8) $\left(R_{C}=1 / M_{C}\right.$, if $\left.m_{k}=1\right)$. Then, this value is compared with the total resistance seen between source and drain also at the cut-off region:

$$
R_{\text {Total }}=R_{\text {channel }}+R_{C}=\frac{V_{D}}{I_{B}}
$$

where $R_{\text {channel }}$ is the equivalent resistance of the intrinsic channel in the subthreshold region. This comparison is carried out at different light intensities, with the transistor in deep subthresh-

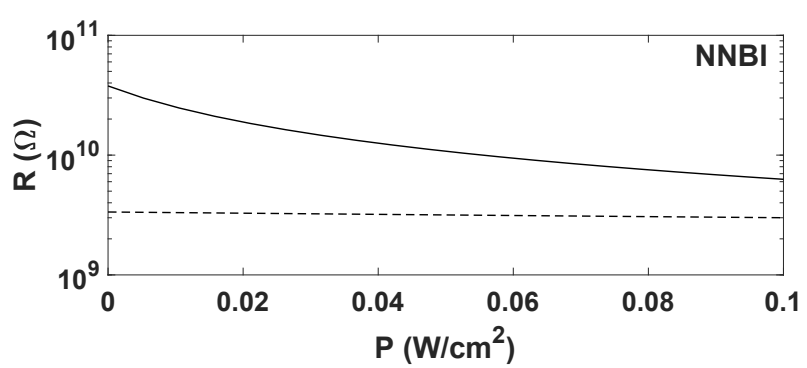

(a)

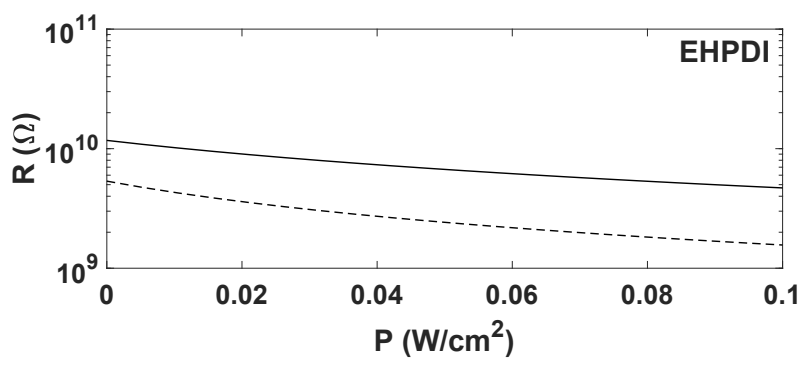

(b)

Figure 8: Evolution with illumination of the total resistance between source and drain (contact resistance plus channel resistance) (solid line) and the contact resistance (dashed line) determined in the subthreshold region $\left(V_{G}=-80 \mathrm{~V}\right.$, $V_{D}=80 \mathrm{~V}$ ) for (a) NBBI and (b) EHPDI based OPTs.

old $\left(V_{G}=-80 \mathrm{~V}\right)$ and the value of $V_{D}$ is the one used in the transfer characteristics of Figs. 3 and 6.

The solid lines of Figs. $8 \mathrm{a}$ and $8 \mathrm{~b}$ show the total resistance $R_{\text {Total }}$ of the NBBI and EHPDI transistors, respectively, as a function of the light intensity, introducing the experimental values of $I_{B}$ and $V_{D}$ in (16). The value of the total resistance is one order of magnitude greater than the value of the contact resistance calculated from (8) (dashed lines). A typical contact length below $100 \mathrm{~nm}$, as deduced by noncontact scanningprobe techniques [23], would produce a lower contact resistance. Thus, morphological differences between the bulk and contact region must play an important role in increasing the value of the contact resistance. Also, it is remarkable how the contact resistances decrease with illumination. In Fig. $8 \mathrm{a}, R_{C}$ decreases around $12 \%$ from dark to illumination (hard to distinguish in the figure). However, the decrease of $R_{C}$ in Fig. $8 \mathrm{~b}$ is greater and follows the same trend as the total resistance. This comparison shows how sensitive our model is to determining the value of the contact resistance even in the cut-off regime of the transistor.

\section{Conclusion}

A model that describes how the contact region of organic phototransitors evolves with the incident optical power has been proposed. This model was included into a compact model for the current-voltage characteristics of OPTs, valid for all operation regions. The compact model works in combination with an evolutionary parameter extraction procedure that allows to reach parameter sets with physical meaning. The model reproduces published experimental current-voltage curves in OPTs 
at different illumination conditions. Our study shows that the contact region of phototransistors is very sensitive to the photovoltaic and photoconductive effects. Therefore, compact models for phototransistors must include not only information about the contact region, but also how the contact region depends on the illumination intensity.

While contact effect in organic thin film transistor persists as a problem to be solved in these devices, models must incorporate this effect. In this regard, the way other OTFT based sensors react to different physical or chemical variables can be better understood with a better description on how the contact region depends on such variables, being this a topic for a future work.

[1] P. Gu, Y. Yao, L. Feng, S. Niu, H. Dong, Recent advances in polymer phototransistors, Polym. Chem. 6 (46) (2015) 7933-7944.

[2] Organic Electronics Association, et al., OE-A roadmap for organic and printed electronics, White Paper (2017).

[3] K. Takimiya, M. Nakano, H. Sugino, I. Osaka, Design and elaboration of organic molecules for high field-effect-mobility semiconductors, Synth. Met. 217 (2016) 68-78.

[4] T. Uemura, C. Rolin, T.-H. Ke, P. Fesenko, J. Genoe, P. Heremans, J. Takeya, On the extraction of charge carrier mobility in high-mobility organic transistors, Adv. Mater. 28 (1) (2016) 151-155.

[5] O. Marinov, M. J. Deen, B. Iñiguez, Performance of organic thin-film transistors, J. Vac. Sci. Technol., B 24 (4) (2006) 1728.

[6] K. Fukuda, Y. Takeda, M. Mizukami, D. Kumaki, S. Tokito, Fully solution-processed flexible organic thin film transistor arrays with high mobility and exceptional uniformity, Scientific Reports 4 (2014) 3947.

[7] R. Picos, E. Garcia, M. Estrada, A. Cerdeira, B. Iñiguez, Effect of process variations on an OTFT compact model parameters, Int. J. High Speed Electron. Syst. 20 (04) (2011) 815-828.

[8] J. Carrabina, M. Mashayekhi, J. Pallarès, L. Terés, Inkjet-configurable gate arrays (IGA), IEEE Trans. Emerging Top. Comput. 5 (2) (2017) 238 246.

[9] T.-Y. Chu, Z. Zhang, A. Dadvand, C. Py, S. Lang, Y. Tao, Direct writing of inkjet-printed short channel organic thin film transistors, Org. Electron. 51 (2017) 485-489.

[10] Z. Qi, J. Cao, H. Li, L. Ding, J. Wang, High-performance thermally stable organic phototransistors based on PSeTPTI/PC61 bm for visible and ultraviolet photodetection, Adv. Funct. Mater. 25 (21) (2015) 3138-3146.

[11] J. Zhong, X. Wu, S. Lan, Y. Fang, H. Chen, T. Guo, High performance flexible organic phototransistors with ultrashort channel length, ACS Photonics 5 (9) (2018) 3712-3722.

[12] X. Wang, K. Wasapinyokul, W. D. Tan, R. Rawcliffe, A. J. Campbell, D. D. C. Bradley, Device physics of highly sensitive thin film polyfluorene copolymer organic phototransistors, J. Appl. Phys. 107 (2) (2010) 024509 .

[13] Y. Peng, W. Lv, B. Yao, J. Xie, T. Yang, G. Fan, D. Chen, P. Gao, M. Zhou, Y. Wang, Improved performance of photosensitive field-effect transistors based on palladium phthalocyanine by utilizing al as source and drain electrodes, IEEE Trans. Electron. Dev. 60 (3) (2013) 1208-1212.

[14] Y. Lei, N. Li, W.-K. E. Chan, B. S. Ong, F. Zhu, Highly sensitive near infrared organic phototransistors based on conjugated polymer nanowire networks, Org. Electron. 48 (2017) 12-18.

[15] B. Yao, Y. Li, Z. Fang, Y. Tan, S. Liu, Y. Peng, H. Xu, Investigation of the source-drain electrodes/the active layer contact-effect on the performance of organic phototransistor, Synth. Met. 233 (2017) 58-62.

[16] C. S. S. Sangeeth, P. Stadler, S. Schaur, N. S. Sariciftci, R. Menon, Interfaces and traps in pentacene field-effect transistor, J. Appl. Phys. 108 (11) (2010) 113703.

[17] W. Boukhili, C. Tozlu, M. Mahdouani, S. Erten-Ela, R. Bourguiga, Illumination and dipole layer effects on the density of state distribution in n-type organic thin film phototransistors based on naphthalene bis-benzimidazole: Experiment and modeling, Microelectron. Eng. 179 (2017) 37-47.

[18] X. Liu, M. Zhang, G. Dong, X. Zhang, Y. Wang, L. Duan, L. Wang, Y. Qiu, The effect of oxygen content on the performance of low-voltage organic phototransistor memory, Org. Electron. 15 (7) (2014) 1664-1671.
[19] Y. Peng, W. Lv, B. Yao, G. Fan, D. Chen, P. Gao, M. Zhou, Y. Wang, High performance near infrared photosensitive organic field-effect transistors realized by an organic hybrid planar-bulk heterojunction, Org. Electron. 14 (4) (2013) 1045-1051.

[20] P. V. Necliudov, M. S. Shur, D. J. Gundlach, T. N. Jackson, Modeling of organic thin film transistors of different designs, J. Appl. Phys. 88 (11) (2000) 6594-6597.

[21] O. Marinov, M. J. Deen, B. Iñiguez, Charge transport in organic and polymer thin-film transistors: recent issues, IEE Proceedings - Circuits, Devices and Systems 152 (3) (2005) 189.

[22] D. Gundlach, L. Zhou, J. Nichols, T. Jackson, P. Necliudov, M. Shur, An experimental study of contact effects in organic thin film transistors, J. Appl. Phys. 100 (2) (2006) 024509.

[23] M. J. Deen, M. Kazemeini, S. Holdcroft, Contact effects and extraction of intrinsic parameters in poly(3-alkylthiophene) thin film field-effect transistors, J. Appl. Phys. 103 (12) (2008) 124509.

[24] W. Liu, C. Hu, BSIM4 and MOSFET modeling for IC simulation, World Scientific, 2011

[25] O. Marinov, M. J. Deen, U. Zschieschang, H. Klauk, Organic thin-film transistors: Part I-compact dc modeling, IEEE Trans. Electron. Dev. 56 (2009) 2952-2961.

[26] M. J. Deen, O. Marinov, U. Zschieschang, H. Klauk, Organic Thin-Film Transistors: Part II. Parameter Extraction, IEEE Trans. Electron. Dev. 56 (12) (2009) $2962-2968$.

[27] J. A. Jiménez Tejada, J. A. López Villanueva, P. López-Varo, K. M. Awawdeh, M. J. Deen, Compact modeling and contact effects in organic transistors, IEEE Trans. Electron. Dev. 61 (2) (2014) 266-277.

[28] F. Torricelli, M. Ghittorelli, M. Rapisarda, A. Valletta, L. Mariucci, S. Jacob, R. Coppard, E. Cantatore, Z. Kovács-Vajna, L. Colalongo, Unified drain-current model of complementary p- and n-type OTFTs, Org. Electron. 22 (Supplement C) (2015) 5-11.

[29] A. Valletta, M. Rapisarda, S. Calvi, G. Fortunato, M. Frasca, G. Maira, A. Ciccazzo, L. Mariucci, A DC and small signal AC model for organic thin film transistors including contact effects and non quasi static regime, Org. Electron. 41 (Supplement C) (2017) 345-354.

[30] A. Romero, J. González, J. Jiménez-Tejada, Constrained many-objective evolutionary extraction procedure for an OTFT compact model including contact effects., in: $12^{\text {th }}$ Spanish Conference on Electron Devices, 2018.

[31] M. J. Deen, M. H. Kazemeini, Y. M. Haddara, J. Yu, G. Vamvounis, S. Holdcroft, W. Woods, Electrical characterization of polymer-based FETs fabricated by spin-coating poly(3-alkylthiophene)s, IEEE Trans. Electron. Dev. 51 (11) (2004) 1892-1901.

[32] P. López-Varo, J. A. Jiménez Tejada, O. Marinov, J. Carceller, C. Chen, M. Deen, Boundary condition model for the simulation of organic solar cells, Org. Electron. 48 (2017) 85-95.

[33] O. Marinov, M. J. Deen, R. Datars, Compact modeling of charge carrier mobility in organic thin-film transistors, J. Appl. Phys. 106 (6) (2009) 064501 .

[34] P. Lara Bullejos, J. A. Jiménez Tejada, F. M. Gómez-Campos, M. J. Deen, O. Marinov, Evaluation of the charge density in the contact region of organic thin film transistors, J. Appl. Phys. 106 (9) (2009) 094503.

[35] Y. Tsividis, Operation and Modeling of the MOS Transistor, 2nd Edition, New York: McGraw-Hill, 1999.

[36] M. Jacunski, M. Shur, A. Owusu, T. Ytterdal, M. Hack, B. Iniguez, A short-channel DC SPICE model for polysilicon thin-film transistors including temperature effects, IEEE Trans. Electron. Dev. 46 (6) (1999) 1146-1158.

[37] M. S. Shur, T. A. Fjeldly, T. Ytterdal, Transistor modeling for the vdsm era, in: Quality Electronic Design, 2000. ISQED 2000. Proceedings. IEEE 2000 First International Symposium on, IEEE, 2000, pp. 37-43.

[38] J. A. Jiménez Tejada, K. M. Awawdeh, J. A. López Villanueva, J. E. Carceller, M. J. Deen, N. B. Chaure, T. Basova, A. K. Ray, Contact effects in compact models of organic thin film transistors: Application to zinc phthalocyanine-based transistors, Org. Electron. 12 (5) (2011) 832-842.

[39] O. Marinov, M. J. Deen, C. Feng, Y. Wu, Precise parameter extraction technique for organic thin-film transistors operating in the linear regime, J. Appl. Phys. 115 (3) (2014) 034506.

[40] J. A. Jiménez Tejada, P. López-Varo, A. N. Cammidge, I. Chambrier, M. J. Cook, N. B. Chaure, A. K. Ray, Compact modeling of organic thin-film transistors with solution processed octadecyl substituted tetrabenzotriazaporphyrin as an active layer, IEEE Trans. Electron. Dev. 64 (6) 
(2017) 2629-2634.

[41] J. A. Jiménez Tejada, P. Lopez-Varo, N. B. Chaure, I. Chambrier, A. N. Cammidge, M. J. Cook, A. Jafari-Fini, A. K. Ray, Organic thin film transistors using a liquid crystalline palladium phthalocyanine as active layer, J. Appl. Phys. 123 (11) (2018) 115501.

[42] M. Fayez, K. M. Morsi, M. N. Sabry, OTFTs compact models: analysis, comparison, and insights, IET Circuits Devices Syst. 11 (5) (2017) 409420.

[43] K. Awawdeh, J. J. Tejada, P. L. Varo, J. L. Villanueva, F. G. Campos, M. Deen, Characterization of organic thin film transistors with hysteresis and contact effects, Org. Electron. 14 (12) (2013) 3286-3296.

[44] K. D. Holland, A. U. Alam, N. Paydavosi, M. Wong, C. M. Rogers, S. Rizwan, D. Kienle, M. Vaidyanathan, Impact of contact resistance on the $f_{t}$ and $f_{\max }$ of graphene versus $\mathrm{MoS}_{2}$ transistors, IEEE Trans. Nanotechnol. 16 (1) (2017) 94-106.

[45] S. V. Suryavanshi, E. Pop, S2DS: Physics-based compact model for circuit simulation of two-dimensional semiconductor devices including nonidealities, J. Appl. Phys. 120 (22) (2016) 224503.

[46] A. Romero, J. González, R. Picos, M. Deen, J. Jiménez-Tejada, Evolutionary parameter extraction for an organic TFT compact model including contact effects, Org. Electron. 61 (2018) 242 - 253.

[47] R. Liguori, W. Sheets, A. Facchetti, A. Rubino, Light- and bias-induced effects in pentacene-based thin film phototransistors with a photocurable polymer dielectric, Org. Electron. 28 (2016) 147-154.

[48] H.-S. Kang, C.-S. Choi, W.-Y. Choi, D.-H. Kim, K.-S. Seo, Characterization of phototransistor internal gain in metamorphic high-electronmobility transistors, Appl. Phys. Lett. 84 (19) (2004) 3780-3782.

[49] M. Hamilton, S. Martin, J. Kanicki, Thin-film organic polymer phototransistors, IEEE Trans. Electron. Dev. 51 (6) (2004) 877-885.

[50] S. Mansouri, A. Jouili, L. E. Mir, A. A. Al-Ghamdi, F. Yakuphanoglu, Studies of photosensitivity and photo-induced negative differential resistance (NDR) of TIPS-pentacene-poly(3-hexyl)thiophene blend organic thin film transistor, Synth. Met. 207 (2015) 1-12.

[51] J. Milvich, T. Zaki, M. Aghamohammadi, R. Rödel, U. Kraft, H. Klauk, J. N. Burghartz, Flexible low-voltage organic phototransistors based on air-stable dinaphtho[2,3-b:2,,3/-f]thieno[3,2-b]thiophene (DNTT), Org. Electron. 20 (2015) 63-68.

[52] M. Deen, M. Kazemeini, Photosensitive polymer thin-film FETs based on poly(3-octylthiophene), Proceedings of the IEEE 93 (7) (2005) 13121320 .

[53] Y. Takanashi, K. Takahata, Y. Muramoto, Characteristics of InAlAs/InGaAs high electron mobility transistors under $1.3-\mu \mathrm{m}$ laser illumination, IEEE Electron Device Lett. 19 (12) (1998) 472-474.

[54] Y. Takanashi, K. Takahata, Y. Muramoto, Characteristics of InAlAs/InGaAs high-electron-mobility transistors under illumination with modulated light, IEEE Trans. Electron. Dev. 46 (12) (1999) 2271-2277.

[55] C. Y. Chen, Theory of a modulated barrier photodiode, Appl. Phys. Lett. 39 (12) (1981) 979-981.

[56] C. Tozlu, M. Kus, M. Can, M. Ersöz, Solution processed white light photodetector based n, n/-di (2-ethylhexyl)-3,4,9,10-perylene diimide thin film phototransistor, Thin Solid Films 569 (2014) 22-27.

[57] W. Huang, Y.-H. Lin, T. D. Anthopoulos, High speed ultraviolet phototransistors based on an ambipolar fullerene derivative, ACS APPL. MATER. INTER. 10 (12) (2018) 10202-10210.

[58] S. Luke, ECJ evolutionary computation library, available for free at http://cs.gmu.edu/ eclab/projects/ecj/ (1998).

[59] J. González, I. Rojas, J. Ortega, H. Pomares, F. Fernandez, A. Díaz, Multiobjective evolutionary optimization of the size, shape, and position parameters of radial basis function networks for function approximation, IEEE Trans. Neural Networks 14 (6) (2003) 1478-1495.

[60] C. Tozlu, S. Erten-Ela, S. Icli, Photoresponsive n-channel organic field effect transistor based on naphthalene bis-benzimidazole with divinyltetramethyl disiloxane-bis (benzo-cyclobutene) gate insulator, Sens. Actuators, A 161 (1-2) (2010) 46-52.

[61] P. Toman, M. Menšík, W. Bartkowiak, J. Pfleger, Modelling of the charge carrier mobility in disordered linear polymer materials, Phys. Chem. Chem. Phys. 19 (11) (2017) 7760-7771.

[62] E. Kozma, M. Catellani, Perylene diimides based materials for organic solar cells, Dyes Pigm. 98 (1) (2013) 160-179.

[63] C. Li, H. Wonneberger, Perylene imides for organic photovoltaics: Yesterday, today, and tomorrow, Adv. Mater. 24 (5) (2012) 613-636. 